\title{
Anomalous diffusion through coupling to a fractal environment: Microscopic derivation of the "whip-back" effect.
}

\author{
Eric Lutz \\ Max-Planck-Institut für Kernphysik, Postfach 103980, 69029 Heidelberg, Germany
}

(November 3, 2018)

\begin{abstract}
Two models for quantum Brownian motion - the Oscillator Bath (OB) model and the Random Band-Matrix (RBM) model - are compared and a relation between the spectral density function $I(\omega)$ and the variance $\overline{V_{a b}{ }^{2}}$ is established. The extension to a fractal environment is then considered and the microscopic origin of anomalous diffusion is discussed. In particular, it is shown that the asymptotic behavior of the normalized velocity auto-correlation function (VACF) is entirely determined by the band form factor. This allows for a microscopic derivation of the "whip-back" effect.
\end{abstract}

PACS numbers: 05.40.Jc, 05.30.-d

Anomalous diffusion has attracted considerable attention in the last decade (for a review see [1] 3]) and numerous studies have been devoted to its description, mostly at the phenomenological level. Among different approaches are fractional diffusion equations 国 6], Lévy walks [7], fractional Fokker-Planck equations [8 11, nonextensive statistical mechanics 12, quantum Lévy processes 113 and generalized Langevin equations [14. Anomalous diffusion often results from the interaction with a complex, non-homogeneous background. Examples include porous or disordered media [1], chaotic heat baths [15] and also fractal environments [16]. In this Letter we focus on a system coupled to a fractal heat bath and explore the microscopic origin of anomalous diffusion with the help of the RBM model for quantum Brownian motion introduced in Ref. [17]. In order to make a direct comparison between the OB model 18,19 and the RBM model, and to extent the latter to the case of a fractal environment, we first calculate the bath correlation function and investigate the validity of the KuboMartin-Schwinger (KMS) condition. We then relate the VACF to the microscopic band form factor by employing the first dissipation-fluctuation theorem and derive the "whip-back" effect 20.21.

We consider a quantum system $S$ weakly coupled to a heat bath $B$. The coupling is taken linear in the position coordinate $x$ of the system. The composite Hamiltonian is given by

$$
H=H_{S} \otimes \mathbb{1}_{B}+\mathbb{1}_{S} \otimes H_{B}+x \otimes V,
$$

where $H_{S}\left(H_{B}\right)$ is the system (bath) Hamiltonian and $V$ is an operator acting on the bath. It is assumed that at $t=0, S$ and $B$ are uncorrelated, so that the initial total density operator factorizes, $\hat{\rho}(0)=\hat{\rho}_{S}(0) \otimes \hat{\rho}_{B}(0)$, where $\hat{\rho}_{S, B}(t)=\operatorname{tr}_{B, S}[\hat{\rho}(t)]$. It is further assumed that the bath is initially in thermal equilibrium at temperature $T, \hat{\rho}_{B}(0)=Z^{-1} \exp \left(-\beta H_{B}\right)$, with $\beta=(k T)^{-1}$.

In the standard approach to quantum dissipation [18, 19, the bath is modelled by an ensemble of independent harmonic oscillators (mass $m_{i}$, frequency $\omega_{i}$ ) and $V$ is assumed to be linear in the positions $x_{i}$ of the oscillators, $V=\sum_{i} c_{i} x_{i}$, where the $c_{i}$ 's are coupling constants. The coupling to the heat bath is fully characterized by the spectral density function $I(\omega)$, which is defined by

$$
I(\omega)=\pi \sum_{i} \frac{c_{i}^{2}}{2 m_{i} \omega_{i}} \delta\left(\omega-\omega_{i}\right) .
$$

On the other hand, in the random-matrix model the structure of the bath is not specified, in particular there are no environmental oscillators, and $V$ is a centered Gaussian random band-matrix. In this approach the coupling to the bath is uniquely characterized by the second moment $\overline{V_{a b}{ }^{2}}$ of the random interaction. The explicit form used in Ref. [17] reads

$$
\overline{V_{a b}{ }^{2}}=A_{0}\left[\rho\left(\varepsilon_{a}\right) \rho\left(\varepsilon_{b}\right)\right]^{-\frac{1}{2}} \exp \left[-\frac{\left(\varepsilon_{a}-\varepsilon_{b}\right)^{2}}{2 \Delta^{2}}\right] .
$$

Here $\varepsilon_{a}$ 's denote the eigenenergies of the bath Hamiltonian $\left(H_{B}|a\rangle=\varepsilon_{a}|a\rangle\right), A_{0}$ is the strength of the coupling, $\Delta$ the bandwidth and $\rho(\varepsilon)$ is the density of states of the bath. Using the equivalence of the canonical and microcanonical ensembles in the thermodynamical limit, the bath may be parametrized as 17 .

$$
\hat{\rho}_{B}(0)=\left|a^{*}\right\rangle\left\langle a^{*}\right| \quad \text { and } \quad \rho\left(\varepsilon^{*}\right)=\rho_{0} e^{\beta \varepsilon^{*}},
$$

where the state $|a\rangle^{*}$ is defined by the temperature $T$ and $\rho_{0}=Z / \sqrt{\pi k T^{2} C_{V}}\left(C_{V}\right.$ being the heat capacity at constant volume).

In Ref. [17] we have demonstrated that the averaged Markovian master equation for the system obtained from the RBM model is identical to the equation derived starting from the OB model. This shows that the two models, although being completely different in nature, are somehow related (at least at the level of the master equation). It is our aim to make this connection more precise and to directly relate the quantities that define the two models, namely the spectral density function (2) and the variance (3). This will be achieved by evaluating the bath correlation function. 
The bath correlation function is defined as

$$
K(t)=\langle\widetilde{V}(t) \tilde{V}(0)\rangle_{B}
$$

where $\widetilde{V}(t)=\exp \left(i H_{B} t\right) V \exp \left(-i H_{B} t\right)$ and $\langle\ldots\rangle_{B}=$ $\operatorname{tr}_{B}\left[\hat{\rho}_{B}(0) \ldots\right]$ denotes the thermal average. Performing the average over the random-matrix ensemble in Eq. (5) leads to

$$
\bar{K}(t)=\int_{-\infty}^{+\infty} d \varepsilon_{b} \rho\left(\varepsilon_{b}\right) \overline{V_{a b}} e^{i\left(\varepsilon_{a}-\varepsilon_{b}\right) t} .
$$

The above equation shows that $\bar{K}(t)$ is essentially the Fourier transform of the variance $\overline{V_{a b}{ }^{2}}$ with respect to $\varepsilon_{b}$. On the other hand, the correlation function can also be written in terms of the spectral density $I(\omega)$ as

$$
\begin{aligned}
K(t) & =\int_{0}^{\infty} \frac{d \omega}{\pi} I(\omega)\left(\operatorname{coth}\left(\frac{\beta \omega}{2}\right) \cos (\omega t)-i \sin (\omega t)\right) \\
& =K^{\prime}(t)+i K^{\prime \prime}(t)
\end{aligned}
$$

by using the identity of $K(t)$ with the kernel of the influence functional [19]. We see from Eq. (7) that the imaginary part $K^{\prime \prime}(t)$ of the correlation function is simply the Fourier sine transform of the spectral density. Before we make use of this observation to obtain the relation between $I(\omega)$ and $\overline{V_{a b}{ }^{2}}$, let us find the most general form of the variance allowed by the laws of thermodynamics. More specifically, let us determine the conditions under which $\bar{K}(t)$ satisfies the KMS condition, $\bar{K}(-t)=\bar{K}(t-i \beta)$, which is known to define the thermal equilibrium state of the bath [22,23]. To this end, we consider the following form of the variance $(k>0)$,

$$
\overline{V_{a b}{ }^{2}}=A_{0}\left[\rho\left(\varepsilon_{a}\right) \rho\left(\varepsilon_{b}\right)\right]^{-k} \exp \left[-\frac{\left(\varepsilon_{a}-\varepsilon_{b}\right)^{2}}{2 \Delta^{2}}\right] .
$$

Substituting (8) into Eq. (6), we obtain

$$
\bar{K}(t)=\frac{A_{0} \sqrt{2 \pi} \Delta}{\rho\left(\varepsilon_{a}\right)^{2 k-1}} \exp \left[-\frac{\Delta^{2}}{2}(t+i(1-k) \beta)^{2}\right],
$$

where we have replaced the density of states $\rho(\varepsilon)$ by the expression (41). We see from (9) that the KMS condition is only fulfilled for the value $k=1 / 2$ (which is the form we have used so far) and that this is independent of the actual form of the band. This can be understood by the following qualitative argument. Let us write $\left|\varepsilon_{a}-\varepsilon_{b}\right|=$ $n d$ where $d=\rho^{-1}$ is the mean level spacing and let us assume that $n d \ll \Delta$. According to (8), the intensity of the coupling is approximately given by $A_{0} d^{2 k}$. As a result, we observe that the coupling is much stronger for $k=0$ than for $k=1 / 2$ (recall that the spectrum of $B$ is quasi-continuous which means that $d \sim 0$ ). This strong coupling then prevents the bath from being in equilibrium. In the opposite limit $k=1$, the coupling is so weak that the imaginary part $\overline{K^{\prime \prime}}(t)$ vanishes. This implies that there is no dissipation (see Eq. (14)).
The most general expression for the variance which respects the KMS condition is hence of the form

$$
\overline{V_{a b}{ }^{2}}=\left[\rho\left(\varepsilon_{a}\right) \rho\left(\varepsilon_{b}\right)\right]^{-\frac{1}{2}} \mathrm{f}\left(\omega_{a b}=\varepsilon_{b}-\varepsilon_{a}\right),
$$

where we have introduced the (symmetric) band form factor $f(\omega)$. Note that the form (10) ensures the passivity of the heat bath in accordance with the Second Law of thermodynamics 23] (for $k>1$ the damping coefficient defined in Eq. (15) is negative and the bath is clearly active) and also guarantees that the bath remains in equilibrium at all times. Now, by comparing the imaginary parts of Eqs. (6) and (7), we arrive at the relation

$$
I(\omega)=2 \pi \sinh \left(\frac{\beta \omega}{2}\right) \mathrm{f}(\omega) .
$$

This equation provides a link between the two models. A straightforward application of the above formula using the form (3) yields

$$
I(\omega)=2 \pi A_{0} \sinh \left(\frac{\beta \omega}{2}\right) \exp \left[-\frac{\omega^{2}}{2 \Delta^{2}}\right] .
$$

One may also check that the power spectrum of $\overline{K^{\prime}}(t)$ satisfies the fluctuation-dissipation theorem 18, 19.

$$
\overline{K^{\prime}}(\omega)=\operatorname{coth}\left(\frac{\beta \omega}{2}\right) I(\omega) .
$$

At this point, it is useful to introduce the damping kernel

$$
\gamma(t)=\frac{2}{M} \int_{-\infty}^{t} d t^{\prime} \overline{K^{\prime \prime}}\left(t^{\prime}\right) .
$$

In the limit $1 \ll \Delta \ll k T$, (14) is found to be equal to

$$
\gamma(t)=2 \pi \frac{A_{0} \beta}{M} \delta(t)=2 \gamma \delta(t) .
$$

With the help of Eq. (15), Eq. (12) thus reduces to

$$
I(\omega) \sim M \gamma \omega
$$

in the limit $\omega \rightarrow 0$. Clearly, the variance (3) defines Ohmic damping, as expected from the results of Ref. [17]. Let us now turn to the determination of the band form factor in the non-Ohmic case.

In the non-Ohmic regime, the spectral density of the bath obeys $I(\omega) \sim \omega^{\alpha}, \alpha>0$, at low frequencies. For $\alpha$ non-integer, the bath is called fractal [24]. We immediately see that if the band form factor satisfies

$$
\mathrm{f}(\omega) \sim g_{\alpha}|\omega|^{\alpha-1},
$$

in the limit $\omega \rightarrow 0$, then, according to Eq. (11),

$$
I(\omega) \sim \pi \beta \omega \mathrm{f}(\omega) \sim \pi \beta g_{\alpha} \omega^{\alpha}, \quad \omega>0 .
$$




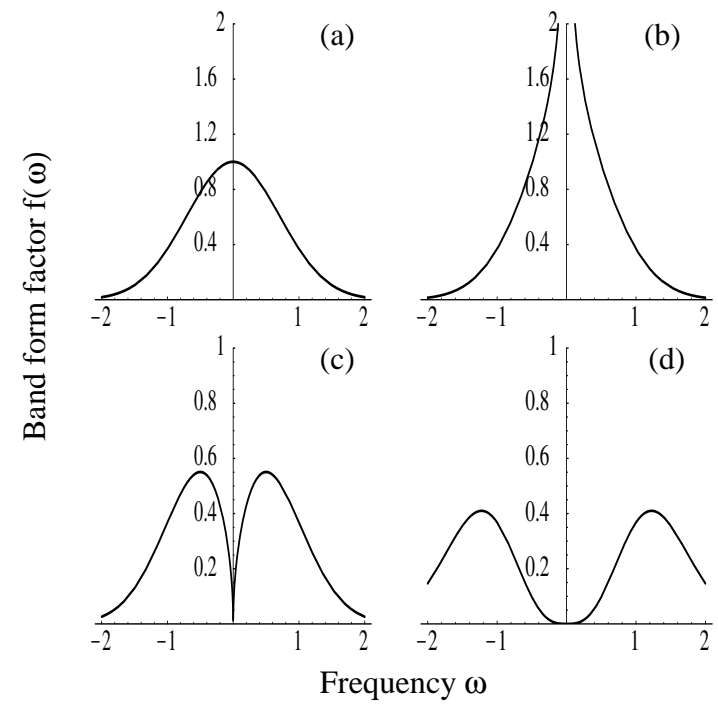

FIG. 1. Band form factor $f(\omega)=|\omega|^{\alpha-1} \exp \left[-\omega^{2}\right]$ for four values of $\alpha$ : (a) $\alpha=1$ (diffusive), (b) $\alpha=2 / 3$ (subdiffusive), (c) $\alpha=3 / 2$ (superdiffusive), (d) $\alpha=4$ (ballistic).

We conclude that the damping regime is determined by the shape of the band at the origin and that the nonOhmic (fractal) regime is the result of a shape of type (17). (Notice that the algebraic singularity appearing in Eq. (6) for $\alpha<1$ can easily be regularized, since the diagonal matrix elements $V_{a a}$ have no physical meaning, by taking Hadamard's finite part $\int_{-\infty}^{\infty} x^{\alpha-1} g(x) d x=$ $\int_{-\infty}^{\infty} x^{\alpha-1}[g(x)-g(0)] d x$, see e.g. 25). Furthermore, it is known that the coupling to a fractal environment gives rise to anomalous diffusion at finite temperature 16, 18. In the long-time limit, the mean-square displacement $s(t)=\left\langle(x(t)-x(0))^{2}\right\rangle_{t}$ for a free damped particle grows like $t^{\alpha}$ for $\alpha<2$ and like $t^{2}$ for $\alpha>2$. This leads to normal diffusion in the Ohmic case $\alpha=1$ and to sub(super)diffusion in the case $\alpha<1(1<\alpha<2)$. For $\alpha>2$, the growth of $s(t)$ is ballistic. These different regimes can be qualitatively understood by looking at the behavior of the band form factor at the origin (see Figure 1). Compared to the diffusive case $\alpha=1$, we observe that subdiffusion is the consequence of the strong enhancement of small energy transfers which occurs for $\alpha<1$ (the absorption by the bath of a given amount of energy necessitates, on average, more steps in the latter case: the bath becomes "stiff" and diffusion is slowed down), while superdiffusion results from the suppression of these small transitions for $1<\alpha<2$. The value $\alpha=2$ is singled out in all anomalous diffusion phenomena. This happens here too. When $\alpha>2$, the derivative at the origin of the function $\omega^{\alpha-1}$ vanishes and the form factor $f(\omega)$ shows a gap around $\omega=0$. As a result, the motion of the free particle is dissipative only as long as its energy is larger than the value of the gap. Once its energy is smaller, the particle behaves, on average, as if it were free (ballistic motion). The foregoing discussion can be made more quantitative and the microscopic origin of anomalous diffusion more transparent by evaluating the VACF of the particle.

We shall now show that the long time behavior of the normalized VACF $C_{v}(t)$ of the system can be written in a simple way in terms of the band form factor. Let us recall that the canonical correlation function of the velocity operator $v$ is defined as [26]

$$
\langle\tilde{v}(0) ; \tilde{v}(t)\rangle=\beta^{-1} \int_{0}^{\beta} d \lambda\langle\tilde{v}(-i \hbar \lambda) \tilde{v}(t)\rangle .
$$

In the limit $\hbar \rightarrow 0$, (19) reduces to the classical correlation function $\langle v(0) v(t)\rangle$. The first fluctuation-dissipation theorem [26]

$$
C_{v}[z]=(z+\gamma[z])^{-1}
$$

then relates the Laplace transform $C_{v}[z]$ of the normalized VACF $C_{v}(t)=\langle\tilde{v}(0) ; \tilde{v}(t)\rangle /\langle\tilde{v}(0) ; \tilde{v}(0)\rangle$ to the Laplace transform of the damping kernel $\gamma(t)$, which is given by [18]

$$
\gamma[z]=\frac{2}{M} \int_{0}^{\infty} \frac{d \omega}{\pi} \frac{I(\omega)}{\omega} \frac{z}{z^{2}+\omega^{2}} .
$$

We further note that in Eq. (21)

$$
\lim _{z \rightarrow 0} \frac{1}{\pi} \frac{z}{z^{2}+\omega^{2}}=\delta(z) .
$$

This gives

$$
\lim _{z \rightarrow 0} \gamma[z]=\frac{\pi \beta}{M} \lim _{z \rightarrow 0} \mathrm{f}(z),
$$

where we have used Eq. (18). Hence, by virtue of the final value theorem [27] (assuming that the limit of $C_{v}(t)$ exists), we can write

$$
\lim _{t \rightarrow \infty} C_{v}(t)=\lim _{z \rightarrow 0}\left(1+\frac{\pi \beta}{M} z^{-1} \mathrm{f}(z)\right)^{-1} .
$$

Eqs. (23) and (24) show that the band form factor governs both the asymptotic behavior of the damping kernel and of the VACF. To be more precise, we shall assume that the VACF is of the form $C_{v}(t) \sim t^{\lambda}$ for large times and employ the following theorem on the asymptotic properties of the Laplace transform of a function $F(t)$ 28]

$$
\begin{aligned}
& \text { If } \quad F(t) \sim c t^{\lambda}, \quad t \rightarrow \infty \\
& \text { then } \quad z F[z] \sim c \Gamma(\lambda+1) z^{-\lambda}, \quad z \rightarrow 0 .
\end{aligned}
$$

This theorem is a consequence of

$$
\int_{0}^{\infty} t^{\lambda} e^{-z t} d t=\Gamma(\lambda+1) z^{-(\lambda+1)} .
$$

In view of Eq. (18), we rewrite the right-hand side of (24) as 


$$
z C_{v}[z] \sim\left(1+\frac{\pi \beta}{M} g_{\alpha} z^{\alpha-2}\right)^{-1}, \quad z \rightarrow 0
$$

Two cases have to be distinguished: $\alpha$ smaller or larger than two. In the case $\alpha<2$, Eq. (27) reduces to

$$
z C_{v}[z] \sim \frac{M k T}{\pi g_{\alpha}} z^{-(\alpha-2)}, \quad z \rightarrow 0 .
$$

By using the theorem (25), we then infer

$$
C_{v}(t) \sim \frac{M k T}{\pi g_{\alpha}} \frac{t^{\alpha-2}}{\Gamma(\alpha-1)}, \quad t \rightarrow \infty .
$$

For $\alpha<1$ (subdiffusion), the normalized VACF has a negative power-law tail (this can be seen by noting that $\Gamma(\alpha-1)=\Gamma(\alpha) /(\alpha-1))$. This negative correlation leads to a incessant change of direction of the velocity of the particle and has been named "whip-back" effect in Ref. 20] (see also Ref. 21]) for this reason. This effect is responsible for the slower diffusion of the particle. In contrast, for $\alpha>1$ (superdiffusion), the VACF possesses a positive tail which means that the particle is more likely to move always in the same direction: this results in a enhanced diffusion. In the Ohmic case $\alpha=1$, the VACF vanishes for large times since $\Gamma(0)=\infty$ and the velocities are therefore uncorrelated. This is of course in agreement with the exact form of the VACF, $C_{v}(t)=\exp (-\gamma t)$, which is obtained by taking the inverse transform of Eq. (20) with $\gamma[z]=\gamma$. On the other hand, in the case $\alpha>2$, we note that $z^{\alpha-2}$ tends to zero in the limit $z \rightarrow 0$. Eq. (27) can therefore be rewritten in the form

$$
z C_{v}[z] \sim 1, \quad z \rightarrow 0,
$$

and we have accordingly

$$
C_{v}(t) \sim 1, \quad t \rightarrow \infty .
$$

The VACF (31) corresponds to ballistic motion.

In conclusion, we have investigated the validity of the KMS condition for the RBM model by calculating the bath correlation function. We have found that the most general form of the variance compatible with the Second Law of thermodynamics is given by Eq. (10). This form guarantees the passivity of the heat bath. We have further derived a relation (Eq. (11)) between the spectral density of the OB model and the band form factor and extended the latter to a fractal environment. We have then discussed the microscopic origin of anomalous diffusion; first qualitatively by examining the form factor at the origin, and second, quantitatively, by expressing the asymptotic behavior of the normalized VACF in terms of the band form factor. This resulted in the derivation of the "whip-back' effect.

We wish to thank H. Grabert for having suggested the direct comparison between the RBM and OB models and H.A. Weidenmüller for useful discussions.
[1] J.-P. Bouchaud and A. Georges, Phys. Rep. 195, 127 (1990).

[2] Lévy Flights and Related Topics in Physics, edited by M.F. Shlesinger, G.M. Zaslavsky, and U. Frisch, Lectures Notes in Physics 450 (Springer, Berlin, 1994).

[3] Anomalous Diffusion. From Basics to Applications, edited by A. Pȩkalski and K. Sznajd-Weron, Lectures Notes in Physics 519 (Springer, Berlin, 1998).

[4] W.R. Schneider and W. Wyss, J. Math. Phys. 30, 134 (1989).

[5] R. Metzler, W.G. Glöckle, and T.F. Nonnenmacher, Physica A 211, 13 (1994).

[6] R. Hilfer, Fractals 3, 549 (1995).

[7] M.F. Shlesinger, B.J. West, and J. Klafter, Phys. Rev. Lett. 58, 1100 (1987).

[8] H.C. Fogedby, Phys. Rev. Lett. 73, 2517 (1994); Phys. Rev. E 58, 1690 (1998); S. Jespersen, R. Metzler, and H.C. Fogedby, Phys. Rev. E 59, 2736 (1999).

[9] G.M. Zaslavsky, Physica D 76, 110 (1994); A.I Saichev, G.M. Zaslavsky, Chaos 7, 753 (1997); G.M. Zaslavsky and B. A. Niyazov, Phys. Rep. 283, 73 (1997).

[10] K.M. Kolwankar and A.D. Gangal, Phys. Rev. Lett. 80, 214 (1998).

[11] R. Metzler, E. Barkai, and J. Klafter, Phys. Rev. Lett. 82, 3563 (1999).

[12] D.H. Zanette and P.A. Alemany, Phys. Rev. Lett. 75, 366 (1995).

[13] D. Kusnezov, A. Bulgac, and G. Do Dang, Phys. Rev. Lett. 82, 1136 (1999).

[14] F. Lillo and R.N. Mantegna, Phys. Rev. Lett. 84, 1061 (2000).

[15] D. Kusnezov, A. Bulgac, and G. Do Dang, Phys. Lett. A 234, 103 (1997).

[16] H. Grabert, P. Schramm, and G.-L. Ingold, Phys. Rev. Lett. 58, 1285 (1987).

[17] E. Lutz and H.A. Weidenmüller, Physica A 267, 354 (1999).

[18] H. Grabert, P. Schramm, and G.-L. Ingold, Phys. Rep. 168, 115 (1988).

[19] U. Weiss, Quantum Dissipative Systems, 2nd ed. (World Scientific, Singapore, 1999).

[20] K. Muralidhar, D. Ramkrishna, H. Nakanishi, and D. Jacobs, Physica A 167, 539 (1990).

[21] K.G. Wang, Phys. Rev. A 45, 833 (1992).

[22] H.S. Spohn and J.L. Lebowitz, Adv. Chem. Phys. 38, 109 (1978).

[23] W. Thirring, A Course in Mathematical Physics, Vol. 4 Quantum Mechanics of Large Systems (Springer, Vien, 1983).

[24] A.J. Leggett et al., Rev. Mod. Phys. 59, 1 (1987).

[25] I.M. Gel'fand and G.E. Shilov, Generalized Functions, Vol. 1 (Academic, New York, 1964).

[26] R. Kubo, Rep. Prog. Phys. 29, 255 (1966); R. Kubo, M. Toda, N. Hashitsume, Statistical Physics II, 2nd ed. (Springer, Berlin, 1991).

[27] J.G. Holbrook, Laplace Transforms for Electronic Engineers, 2nd ed. (Pergamon, Oxford, 1966).

[28] G. Doetsch, Introduction to the Theory and Application of the Laplace Transform, (Springer, Berlin, 1974). 